\title{
Algorithm for detecting polarity of opinions in laptop and restaurant domains
}

\section{Autores}

Jose Silva, Noel Varela, Danelys Cabrera, Omar Lezama, Jesus Varas, Patricia Manco

\begin{abstract}
The easy access to the Internet and the large amounts of information produced on the Web, Artificial Intelligence and more specifically the Natural Language Processing (NLP) provide information extraction mechanisms. The information found on the Internet is presented in most cases in an unstructured way, and examples of this are the social networks, source of access to opinions, products or services that society generates daily in these sites. This information can be a source for the application of the NLP, which is responsible for the automatic detection of feelings expressed in the texts and its classification according to the polarity they have; it is the area of analysis of feelings, also called opinion mining. This paper presents a study for the detection of polarity in a set of user opinions issued to Restaurants in Spanish and English.
\end{abstract}

Palabras clave:

Opinion mining, Supervised learning, Natural Language Processing. 